\title{
High prevalence of TERT promoter mutations in primary squamous cell carcinoma of the urinary bladder
}

\author{
Morgan Cowan ${ }^{1}$, Simeon Springer ${ }^{2,3}$, Doreen Nguyen ${ }^{1}$, Diana Taheri ${ }^{1}$, Gunes Guner ${ }^{1}$, \\ Maria Angelica Mendoza Rodriguez ${ }^{1}$, Yuxuan Wang ${ }^{3}$, Isaac Kinde ${ }^{3}$, \\ Christopher J VandenBussche ${ }^{1}$, Matthew T Olson ${ }^{1}$, Isabela Cunha ${ }^{4}$, Kazutoshi Fujita ${ }^{5}$, \\ Dilek Ertoy $^{6}$, Trinity J Bivalacqua ${ }^{7}$, Kenneth Kinzler ${ }^{2,3}$, Bert Vogelstein ${ }^{2,3}$, George J Netto ${ }^{1,7}$ \\ and Nickolas Papadopoulos 2,3 \\ ${ }^{1}$ Department of Pathology, Johns Hopkins University, Baltimore, MD, USA; ${ }^{2}$ Department of Oncology, Johns \\ Hopkins University, Baltimore, MD, USA; ${ }^{3}$ The Ludwig Center for Cancer Genetics and Sidney Kimmel \\ Comprehensive Cancer Center, Baltimore, MD, USA ${ }^{4}$ AC Camargo Cancer Centre, Sao Paulo, Brazil; \\ ${ }^{5}$ Department of Urology, Osaka University, Osaka, Japan; ${ }^{6}$ Department of Pathology, Hacettepe University, \\ Ankara, Turkey and ${ }^{7}$ Departments of Urology, Johns Hopkins University, Baltimore, MD, USA
}

TERT promoter mutations (TERT-mut) are detectable in the majority of urothelial carcinomas. The detection of TERT-mut in urine is under investigation as a potential urine-based molecular-screening assay for bladder cancer. A small but significant number of bladder carcinomas are pure squamous cell carcinoma. We sought to assess the incidence of TERT-mut in squamous cell carcinoma of the urinary bladder. A retrospective search of the institutional pathology archives yielded 15 cystectomy specimens performed for squamous cell carcinoma (2000-2014). Histologic slides were reviewed by a senior urologic pathologist to confirm the diagnosis and select a representative formalin-fixed paraffin-embedded tissue block for mutational analysis. All cases yielded adequate material for DNA analysis. Sequencing for TERT-mut was performed using previously described SafeSeq technique. We detected TERT-mut in $12 / 15(80 \%)$ of bladder squamous cell carcinomas. TERT promoter mutations, commonly found in conventional urothelial carcinoma, are also highly prevalent in urinary bladder squamous cell carcinoma suggesting a common tumorigenesis and potential utility as a molecular urine-basedscreening assay.

Modern Pathology (2016) 29, 511-515; doi:10.1038/modpathol.2016.53; published online 11 March 2016

Maintenance of telomeres is a critical function for cell survival and continued replication, and one mechanism by which cells maintain telomeres is through the action of an extension complex that adds telomeric repeats to the ends of chromosomes. ${ }^{1}$ This complex includes the protein product of the telomerase reverse transcriptase (TERT) gene (5p15.33). High rates of activating mutations in the upstream promoter of the TERT gene (TERT-mut) have been found in several solid tumor types. ${ }^{2-5}$ Mutations tend to occur in 'hot spots', particularly g.1295228C $>\mathrm{T}$ and g.1295250C $>\mathrm{T}$. These

Correspondence: Dr GJ Netto MD, Department of Pathology, Johns Hopkins Hospital, 401 North Broadway Street, Weinberg 2242, Baltimore, MD 21231, USA.

E-mail: gnetto1@jhmi.edu

Received 19 November 2015; revised 8 February 2016; accepted 9 February 2016; published online 11 March 2016 mutations generate a CCGGAA/T or GGAA/T motif, thereby altering the binding site for Ets transcription factor, thereby increasing TERT promoter activity. ${ }^{2,6,7}$

Our group and others have demonstrated TERT-mut to be the most common genetic alterations in urothelial (transitional cell) carcinoma of the bladder and upper urinary tract. ${ }^{8-13}$ In the study by Kinde et $a,^{12} 66 \%$ of muscle invasive and $74 \%$ of non-muscle invasive bladder lesions were shown to harbor these alterations.

Greater than $90 \%$ of bladder carcinomas are urothelial type. Squamous cell carcinoma, adenocarcinoma, and small cell carcinoma represent the remaining most common types. ${ }^{14,15}$ Recently, we investigated TERT-mut incidence in small cell carcinomas of the urinary bladder. ${ }^{16}$ In the current study, we sought to determine the prevalence of 


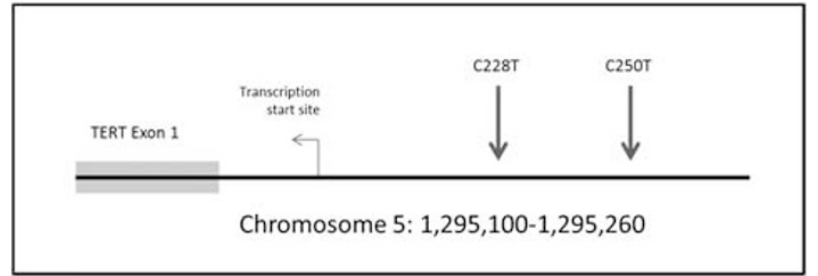

Figure 1 Two mutational 'hotspots are repeatedly seen in the TERT promoter, at position 250 and position228. Both of the mutations are a C-> T base substitution mutation.

TERT-mut in primary squamous cell carcinoma of the urinary bladder.

\section{Materials and methods}

A retrospective review was performed and the archives of a single institution were searched for cases of pure squamous cell carcinoma primary to the bladder treated by cystectomy, cystoprostatectomy, or other radical excision between the years 2000 and 2014. To ensure the inclusion of only 'pure' squamous cell carcinoma cases that would satisfy the World Health Organization/International Society of Urologic Pathology (WHO/ISUP 2004) definition of the entity, we limited our study to cystectomy and cystoprostatectomy specimens. All histologic slides were reviewed by a senior urologic pathologist to confirm the diagnosis of squamous cell carcinoma and select a representative tumor formalin-fixed paraffin-embedded block for mutational analysis. A total of 15 cases were included in the study based on the availability of formalin-fixed paraffin-embedded blocks with sufficient tumor for sampling. The tumor areas were cored with a sterile 16-gauge needle and the fraction of neoplastic cells was estimated from adjacent sections. The cores were placed in $1.5 \mathrm{ml}$ sterile tubes for DNA purification.

Following DNA purification, samples were analyzed with Safe-SeqS, a sequencing error-reduction technology described previously, ${ }^{17}$ which better discriminates genuine TERT-mut from artefactual sequencing variants introduced during the sequencing process. Safe-SeqS amplification primers were designed to amplify segments containing the region of the TERT promoter previously shown to harbor mutations in melanomas and other tumors (Figure 1). ${ }^{12}$ The forward and reverse amplification primers contained the TERT-specific sequences at their $3^{\prime}$ ends and a universal priming site at their $5^{\prime}$ end. The reverse primer additionally contained a 14-base unique identifier comprised of 14 degenerate $\mathrm{N}$ bases (equal likelihood of being an $\mathrm{A}, \mathrm{C}$, $\mathrm{T}$, or $\mathrm{G}$ ) between the universal priming site and gene-specific sequences. The sequences of the forward and reverse primers were either $5^{\prime}$-CACACAGGAAACAGCTAT GACCATGGGCCGCGGAAAGGAAG and 5'-CGACG
TAAAACGACGGCCAGTNNNNNNNNNNNNNNCG TCCTGCCCCTTCACC, or 5'CACACAGGAAACAGC TATGACCATGGCGGAAAGGAAAGGGAG and 5'-CG ACGTAAAACGACGGCCAGTNNNNNNNNNNNNN NCCGTCCCGACCCCTC. PCR products were purified with AMPure and sequenced on a MiSeq instrument. Data were analyzed as previously described. ${ }^{17}$ Tumor samples were considered positive if the fraction of mutations exceeded $1 \%$ of alleles (which was a frequency at least $5 \times$ higher than found in control DNA templates). All sequencing assays scored as positive were confirmed in at least one additional, independent PCR and sequencing experiment.

Two sets of negative control samples were also analyzed. Ninety-four peripheral blood samples from healthy population were tested for TERT-mut as negative PCR procedure controls. Eight formalinfixed paraffin-embedded benign transurethral bladder biopsy samples were also used as negative tissue controls.

\section{Results}

Patient Demographics, Clinicopathologic Features, and Outcome

This cohort included six females and nine males. The mean patient age was 65 years (range 51-83). None of the patients had associated schistosomiasis. The tumors were primarily centered in the bladder wall (12 patients) with the remaining in the trigone, bladder neck urethral region, and bladder dome. of note, one patient had a history of extrophy of the bladder and had a tumor that extended from the bladder wall to involve the dermis of the overlying abdominal wall. Two patients (13\%) had lymph node metastases identified at the time of resection. Mean follow up time was 1564 days with a median follow up interval of 981 days (range 22-5459 days). Two patients $(13 \%)$ died from disease at 337 and 169 days post surgery, the remaining 13 patients are presently disease free (Table 1). Morphologically, all tumors demonstrated typical feature of conventional squamous cell carcinoma. Papillary architecture was observed in one squamous cell carcinoma, and focal sarcomatoid features were found in another case.

\section{TERT Promoter Analysis}

TERT-mut were identified in 12 out of 15 (80\%) cases of squamous cell carcinoma. The majority of these $(83 \%)$ were the conventional morphology, however both the papillary squamous cell carcinoma and squamous cell carcinoma with sarcomatoid features also had TERT-mut. Of all the patients with TERT-mut, $2(17 \%)$ were g.1295250C $>\mathrm{T}$ and the remaining $10(83 \%)$ were a g.1295228C $>\mathrm{T}$ alteration (Table 2). 
Table 1 Patient demographics, clinicopathologic features, and outcome

\begin{tabular}{|c|c|c|c|c|c|c|c|c|c|}
\hline Case & Age & Gender & Histologic type & $\begin{array}{l}\text { Tumor } \\
\text { grade }^{\text {a }}\end{array}$ & Tumor location & Tumor stage & Recurrences & Progression & Outcome ${ }^{\mathrm{b}}$ \\
\hline 1 & 51 & $\mathrm{~F}$ & Conventional & G3 & Bladder wall & T3aNo & No & No & NED \\
\hline 2 & 73 & $\mathrm{M}$ & Conventional & G3 & Bladder wall & T2No & No & No & UNK \\
\hline 3 & 71 & $\mathrm{M}$ & Conventional & G3 & Bladder wall & T3aNo & No & No & UNK \\
\hline 4 & 53 & $\mathrm{~F}$ & Conventional & G3 & $\begin{array}{l}\text { Bladder wall and } \\
\text { skin (extrophy) }\end{array}$ & T4bNX & No & No & NED \\
\hline 5 & 53 & $\mathrm{M}$ & Conventional & G2 & Bladder trigone & T3aNo & Yes & Yes & DOD \\
\hline 6 & 79 & $\mathrm{~F}$ & Conventional & G2 & Bladder wall & T3No & No & No & NED \\
\hline 7 & 73 & $\mathrm{~F}$ & Conventional & $\mathrm{G} 2$ & Bladder wall & $\mathrm{T} 3 \mathrm{Nx}$ & No & No & NED \\
\hline 8 & 56 & $\mathrm{M}$ & Conventional & G2 & Bladder wall & T3bN2 & No & No & NED \\
\hline 9 & 51 & $\mathrm{~F}$ & Conventional & G3 & Bladder wall & T4NX & No & No & NED \\
\hline 10 & 72 & $\mathrm{M}$ & Conventional & $\mathrm{G} 2$ & Bladder wall & T2bNo & No & No & NED \\
\hline 11 & 63 & $\mathrm{~F}$ & Conventional & $\mathrm{G} 2$ & Bladder wall & T3aNo & No & No & NED \\
\hline 12 & 70 & $\mathrm{M}$ & $\begin{array}{l}\text { Papillary squamous } \\
\text { cell carcinoma }\end{array}$ & G3 & Bladder wall & T3bN2 & No & No & NED \\
\hline 13 & 73 & $\mathrm{M}$ & $\begin{array}{l}\text { Squamous cell } \\
\text { carcinoma with focal } \\
\text { sarcomatoid features }\end{array}$ & G1 & Bladder dome & T1NX & No & No & NED \\
\hline 14 & 54 & $\mathrm{M}$ & Conventional & $\mathrm{G} 2$ & $\begin{array}{l}\text { Bladder trigone and } \\
\text { bulbar urethra }\end{array}$ & T2No & No & No & NED \\
\hline 15 & 83 & $\mathrm{M}$ & Conventional & G2 & Bladder wall & T3NX & Yes & Yes & DOD \\
\hline
\end{tabular}

${ }^{\mathrm{a}}$ Tumor grade: well differentiated (G1), moderately differentiated (G2), poorly differentiated (G3). ${ }^{\mathrm{b}}$ Outcome: died of disease (DOD), died with disease (DWD), alive with disease (AWD), no evidence of disease (NED), unknown (UNK).

Table 2 TERT promoter mutations in squamous cell carcinoma by histologic type

\begin{tabular}{|c|c|c|c|}
\hline \multirow{2}{*}{ Histologic type } & \multirow{2}{*}{ Mutation } & \multicolumn{2}{|c|}{ Mutation type } \\
\hline & & TERT g.1295250C $>T$ & TERT g.1295228T $>C$ \\
\hline Classical $(n=13)$ & $10 / 13(77 \%)$ & $2 / 10(20 \%)$ & $8 / 10(80 \%)$ \\
\hline Papillary $(n=1)$ & $1 / 1(100 \%)$ & $0 / 1$ & $1 / 1(100 \%)$ \\
\hline Sarcomatoid features $(n=1)$ & $1 / 1(100 \%)$ & $0 / 1$ & $1 / 1(100 \%)$ \\
\hline All $(n=15)$ & $12 / 15(80 \%)$ & $2 / 12(17 \%)$ & $10 / 12(83 \%)$ \\
\hline
\end{tabular}

All of the blood and formalin-fixed paraffinembedded samples used as negative controls tested negative for TERT-mut.

\section{Discussion}

Bladder carcinoma is the most common malignancy of the urinary tract, and the fourth most common carcinoma in men in the Western world. Among bladder cancers, urothelial carcinoma is by far the most prevalent histologic type, with squamous cell carcinoma and adenocarcinoma making up $<5 \%$ of cases. ${ }^{18}$ Per patient, the cost of bladder cancer management is the highest among all tumors, owing to the need for long-term monitoring with regular cystoscopy, imaging, and urine cytology. ${ }^{19-21}$

TERT-mut were first reported in melanoma. ${ }^{2}$ Subsequently, the same mutations were discovered by our group and others in numerous solid cancers, including urothelial carcinoma, gliomas and hepatocellular carcinoma. ${ }^{3-5}$ High rates of TERT-mut have been conspicuously absent in colorectal and lung carcinomas. ${ }^{3,22}$ More recently, high rates of TERTmut have been demonstrated in small cell carcinoma of the urinary bladder, urothelial carcinoma with squamous differentiation, and nested variant of urothelial carcinoma. ${ }^{13,16,22}$ These consistent genetic alterations, shared by urothelial carcinoma and several of its variants, offer further support for a common oncogenic pathways.

Our study is the first to evaluate the presence of TERT-mut in conventional squamous cell carcinoma of the urinary bladder. As noted above, TERT-mut have been described in several tumor types in the bladder with urothelial differentiation; however, the absence of urothelial differentiation in all 12 cases makes this study novel. All mutations were from previously published hotspots, with the majority of cases $(83 \%)$ having the g.1295228C $>\mathrm{T}$ mutation, whereas the remainder had a g.1295250C $>$ T mutation. Histologically, all 12 TERT-mut-positive tumors were invasive conventional squamous cell carcinoma and with one tumor showing focal 
sarcomatoid differentiation and a second demonstrating a papillary architecture. Of note, one of the three tumors that lacked TERT-mut was the only case in our cohort that was centered in the bulbar urethra. Evaluation of prognostic significance of TERT-mut was not feasible in this limited study.

Our found rate of $80 \%$ prevalence of TERT-mut in squamous cell carcinoma of bladder is comparable to the rate previously demonstrated in conventional urothelial carcinoma including those with only focal squamous differentiation. ${ }^{9,11,12,22}$ This high rate is in stark contrast to the lack of TERT-mut in cervical and pulmonary squamous carcinomas. ${ }^{22}$ If further confirmed, the latter suggests a potential diagnostic role for identifying TERT-mut in assigning a primary urinary tract origin in cases where the differential include secondary involvement of bladder from such sites or cases of unknown primary. Finally, chronic bladder irritation (eg, stones, extrophy, and endemic Schistosomiasis) is associated with higher incidence of bladder squamous cell carcinoma. ${ }^{18}$ To our knowledge, the presence of TERT-mut in squamous cell carcinoma tumors arising in the later setting has not been specifically examined. None of our current patients had associated schistosomiasis. One of our TERT-mut-positive tumors occurred in a patient with a history of bladder extrophy.

In summary, TERT promoter mutations, commonly found in conventional urothelial carcinoma, are also highly prevalent in urinary bladder squamous cell carcinoma, suggesting a common tumorigenesis and potential utility as a molecular urine-based-screening assay.

\section{Acknowledgments}

Supported by grants from The Johns Hopkins Greenberg Bladder Cancer Institute, The Virginia and DK Ludwig Fund for Cancer Research, The Commonwealth Fund, The Conrad R Hilton Foundation, and The Sol Goldman Sequencing Facility at Johns Hopkins.

\section{Disclosure/conflict of interest}

KWK, NP, and BV are founders of Personal Genome Diagnostics, PapGene, and advise Sysmex-Inostics. These companies and others have licensed technologies from Johns Hopkins, of which BV, KWK, and NP are inventors and receive royalties from these licenses. The terms of these arrangements are being managed by the university in accordance with its conflict of interest policies.

\section{References}

1 Lu W, Zhang Y, Liu D et al. Telomeres-structure, function, and regulation. Exp Cell Res 2013;319: 133-141.
2 Huang FW, Hodis E, Xu MJ et al. Highly recurrent TERT promoter mutations in human melanoma. Science 2013;339:957-959.

3 Killela PJ, Reitman ZJ, Jiao Y et al. TERT promoter mutations occur frequently in gliomas and a subset of tumors derived from cells with low rates of selfrenewal. Proc Natl Acad Sci USA 2013;110: 6021-6026.

4 Scott G a, Laughlin TS, Rothberg PG. Mutations of the TERT promoter are common in basal cell carcinoma and squamous cell carcinoma. Mod Pathol 2014;27: $516-523$.

5 Qu Y, Shi L, Wang D et al. Low frequency of TERT promoter mutations in a large cohort of gallbladder and gastric cancers. Int J Cancer 2014;134:2993-2994.

6 Horn S, Figl A, Rachakonda PS et al. TERT promoter mutations in familial and sporadic melanoma. Science 2013;339:959-961.

7 Huang D-S, Wang Z, He X-J et al. Recurrent TERT promoter mutations identified in a large-scale study of multiple tumour types are associated with increased TERT expression and telomerase activation. Eur J Cancer 2015;51:969-976.

8 Allory Y, Beukers W, Sagrera A et al. Telomerase reverse transcriptase promoter mutations in bladder cancer: high frequency across stages, detection in urine, and lack of association with outcome. Eur Urol 2014;65: 360-366.

$9 \mathrm{Wu} \mathrm{S}$, Huang $\mathrm{P}$, Li C et al. Telomerase reverse transcriptase gene promoter mutations help discern the origin of urogenital tumors: a genomic and molecular study. Eur Urol 2014;65:274-277.

10 Rachakonda PS, Hosen I, de Verdier PJ et al. TERT promoter mutations in bladder cancer affect patient survival and disease recurrence through modification by a common polymorphism. Proc Natl Acad Sci USA 2013;110:17426-17431.

11 Borah S, Xi L, Zaug AJ et al. TERT promoter mutations and telomerase reactivation in urothelial cancer. Science 2015;347:1006-1010.

12 Kinde I, Munari E, Faraj SF et al. TERT promoter mutations occur early in urothelial neoplasia and are biomarkers of early disease and disease recurrence in urine. Cancer Res 2013;73:7162-7167.

13 Zhong M, Tian W, Zhuge J et al. Distinguishing nested variants of urothelial carcinoma from benign mimickers by TERT promoter mutation. Am J Surg Pathol 2015;39:127-131.

14 Montironi R, Lopez-Beltran A. The 2004 WHO classification of bladder tumors: a summary and commentary. Int J Surg Pathol 2005;13:143-153.

15 Lopez-Beltran A, Sauter G, Gasser T et al. Tumors of the urinary system. In: Eble JN, Sauter G JI; E, Sesterhenn I (eds). Pathol Genet Tumours Urin Syst Male Genit Organs. Lyon, France: IARC Press, 2004, pp 89-123.

16 Zheng X, Zhuge J, Bezerra SM et al. High frequency of TERT promoter mutation in small cell carcinoma of bladder, but not in small cell carcinoma of other origins. J Hematol Oncol 2014;7:47.

17 Kinde I, Wu J, Papadopoulos $\mathrm{N}$ et al. Detection and quantification of rare mutations with massively parallel sequencing. Proc Natl Acad Sci USA 2011;108: 9530-9535.

18 Cohen SM, Shirai T, Steineck G. Epidemiology and etiology of premalignant and malignant urothelial changes. Scand J Urol Nephrol Suppl 2000;105-115. 
19 Anastasiadis A, Cordeiro E, Bus MT et al. Follow-up procedures for non-muscle-invasive bladder cancer: an update. Expert Rev Anticancer Ther 2012;12: 1229-1241.

20 Anastasiadis A, de Reijke TM. Best practice in the treatment of nonmuscle invasive bladder cancer. Ther Adv Urol 2012;4:13-32.
21 Avritscher EBC, Cooksley CD, Grossman HB et al. Clinical model of lifetime cost of treating bladder cancer and associated complications. Urology 2006;68:549-553.

22 Cheng KA, Kurtis B, Babayeva S et al. Heterogeneity of TERT promoter mutations status in squamous cell carcinomas of different anatomical sites. Ann Diagn Pathol 2015;19:146-148. 